\title{
Performance Analysis for Different Simulation Scenarios for DSDV, DSR and AODV MANET Routing Protocols using NS2 simulator
}

${ }^{\mathrm{a}}$ Kumar Neeraj ${ }^{\mathrm{B}}$ G.SAMPATH KUMAR ${ }^{\mathrm{c}}$ M.KASHYAP CHANDRA ${ }^{\mathrm{d}}$ M.BHARATH KUMAR

${ }^{\text {a }}$ Assistant professor ${ }^{\text {bcd }}$ Student members

abcd Electronics and Communication Engineering Department Anurag Group of Institutions, Hyderabad, Telangana, India.

\section{Abstract}

The protocol selection is one of the most important selection factor in designing any wireless network system . In Mobile Ad-hoc Network (MANET) the chosen protocol should be best as far as information conveyance and information uprightness. Because of this reason, the execution investigation of the conventions is the significant advance before choosing a specific convention. In this paper, we done the execution investigation on Ad-hoc On-request Distance Vector (AODV), Dynamic Source Routing (DSR) and Destination Sequenced Distance Vector (DSDV) conventions utilizing NS2 test system. The deferral, throughput, control overhead and bundle conveyance proportion are the four basic measures utilized for the examination of the execution of above conventions. These execution examinations will be useful for discovering QoS for MANETS.

Keywords: - MANET, QoS, AODV, DSR, DSDV, NS2

\section{INTRODUCTION}

In the current years there has been a gigantic extension in the demand of PCs, note pads, PDA and PDAs. These gadgets are equipped with gigabytes of disk memory, high determination of colour display, pointing gadgets and wireless communication adapters with the facility to work for long times. Among this innovative development, numerous analysts are occupied with investigating the field of Mobile Ad hoc Networks (MANETs). This sort of system is a gathering of self- sufficient wireless mobile nodes system. This dynamic nature of MANET can makes the scheme of being connected "anywhere and at any time" into reality.

There is a need of different protocols and performance of various routing protocols. In the recent years many researches and studies have examined MANET's standard routing protocols; DSDV, AODV and DSR; while focusing on certain criteria over others. Ad-hoc networks comprise of hosts interconnected by switches without a settled foundation and can be organized powerfully.

Considerable work has been done in the development of routing protocols in different types of ad hoc networks like MANETs, WMNs, WSNs, and VANETS etc [1]. The principle vision of MANET is to help vigorous and proficient task in remote systems by joining steering functionalities at every versatile hub. For such outlining parts of specially appointed systems Routing-based approach, Information-theoretic approach, Dynamic control approach or Gametheoretic approach has been executed. [2]. In MANET to help versatile registering a portable 
DOI: https://dx.doi.org/10.26808/rs.ca.i8v2.04

host must have the capacity to speak with other portable hosts which may not exist in it's radio transmission go. Routing Protocols should perform four critical capacities as assurance of system topology, keeping up arrange network, transmission booking and channel task, and parcel steering. Steering conventions in MANETs were created in light of the plan objectives of insignificant control overhead, negligible handling overhead, multi jump directing ability, and dynamic topology upkeep and circle avoidance [3]. Classification of Routing protocols in MANETs should be possible on directing technique savvy or system structure shrewd. As indicated by steering system the directing conventions can be classified as table-driven or proactive and source-started or receptive or on-request directing. Every one of these kinds of conventions carries on diversely on various remote conditions. Subsequently the execution investigation of these conventions is an unquestionable requirement assignment to know its conduct and work in that condition. A few variables will influence the general execution of any convention working in a specially appointed system. For instance, hub portability may cause connect disappointments, which contrary affect on steering and nature of administration (QoS) bolster.

The essential target of this paper is to assess and evaluate the impacts of different components that may impact organize execution. While there has been execution investigation of specially appointed systems [4-6], still a portion of the persuasive factor assessment is likewise absent. Again none of these papers have contrasted and DSR as a directing convention and furthermore they have not considered geological system measure into account. The rest of this paper is composed as tails they gives a concise thought on the diverse steering conventions utilized for execution examination, portrays the reproduction philosophy and execution measurements, recreation results and plan investigation took after by the conclusion and future work.

\section{MANET ROUTING PROTOCOLS}

In this segment, we quickly depict the key highlights of the AODV, DSDV, and DSR conventions examined in our reproductions. We likewise portray the specific parameters that we pick while actualizing every convention. Yet, before that the fundamental contrasts in these convention usage lies in the systems they took after as per directing technique based order as receptive and proactive conventions. In Reactive or on-request steering courses are just found when they are really required. Consequently, a hub that needs to send a parcel to another hub, the responsive conventions scans for the course in an on-request premise and builds up an association with transmit and get a bundle. The course revelation normally comprises of system wide flooding of demand message. Conversely, in proactive steering every hub constantly keep up course between combine of hubs. Thus, course creation and upkeep is proficient through some blend of intermittent and occasion activated steering refreshes got from separate vector or connection state technique. Both these methodologies have a few preferences and a few inconveniences and can be dissected from its execution measurements as talked about in next area. In this article, we concentrated on AODV and DSR as receptive convention and DSDV as connection state proactive convention.

\section{A. Ad-hoc On-demand Distance Vector (AODV)}

AODV is a blend of on-request and separation vector i.e. hop to hop routing [7]. At the point when a hub has to know a course to a particular goal it makes a ROUTE REQUEST. Next the course ask for is sent by middle of the road hubs which additionally make a turnaround course for itself for goal. At the point when the demand achieves a hub with course to goal it makes 
DOI: https://dx.doi.org/10.26808/rs.ca.i8v2.04

again a REPLY, which contains the quantity of jumps that are, require achieving the goal. All hubs that take an interest in sending this answer to the source hub make a forward course to goal. This course made from every hub from source to goal is a jump by-bounce state and not the whole course as in source directing.

\section{B. Destination Sequenced Distance Vector (DSDV)}

DSDV is a hop-to-hop distance vector steering convention requiring every hub to intermittently communicate directing updates in light of the possibility of traditional Bellman-Ford Routing calculation [8]. Every hub keeps up a steering table posting the "following bounce" for each reachable goal, number of jumps to achieve goal and the grouping number doled out by goal hub. The grouping number is utilized to recognize stale courses from new ones and in this way stay away from circle arrangement. The stations occasionally transmit their steering tables to their prompt neighbors. A station additionally transmits its steering table if a critical change has happened in its table from the last refresh sent. In this way, the refresh is both time-driven and occasion driven. The directing table updates can be sent in two ways: a "full dump" or an "incremental" update

\section{Dynamic Source Routing (DSR)}

DSR is a straightforward and proficient steering convention planned particularly for use in multihop remote impromptu systems of versatile hubs [10]. It enables hubs to progressively find a source course over different system jumps to any goal in the specially appointed system. Every datum parcel sent at that point conveys in its header the total requested rundown of hubs through which the bundle must pass, permitting parcel directing to be an inconsequentially circle free and evading the requirement for a la mode steering data in the middle hubs through which the bundle is sent. With the incorporation of this source course in the header of every datum bundle, different hubs sending or catching any of the parcels may effectively reserve this directing data for some time later.

\section{SIMULATION METHODOLOGY AND PERFORMANCE METRICS}

\section{A. Simulation Modeling}

Simulation helps in analyzing the performance and behavior of complex networks before implementing it in today's real application. Several network simulators are available, whose output depicts as close as possible to real time implementation. In this work, we used the discrete-event simulator NS2 (version 2.35) and the performance analysis were conducted using AWK script [12].

\section{B. Simulation Methods and parameters}

. The objective of our tests is to look at and evaluate the impacts of different variables and their cooperation's on the general execution of specially appointed systems. Each keep running of the test system acknowledges as information a situation record that portrays the correct movement of every hub utilizing Random Waypoint versatility demonstrate and the correct succession of bundles began by every hub together with correct time at which change in parcel or movement start happens. Consequently, to assess the execution at a specific factor, we consider 10 irregular reproduction rushes to create 10 arbitrary situation designs and the execution of the considered factor is the normal of these 10 yields. In every one of our examinations, we considered five 
DOI: https://dx.doi.org/10.26808/rs.ca.i8v2.04 International Journal of Computer Application (2250-1797)

Issue 8 Volume 2, March-April 2018

example purposes of a specific factor and checked for three distinct conventions i.e. AODV, DSR and DSDV. Subsequently 150 reenactment runs were directed to break down every execution factor for these three conventions. Since our examinations depends on arrange layer qualities so changes in steering technique is just watched whereas different attributes like reception apparatus pick up, transmit control, ground spread model and beneficiary affectability as physical layer qualities, MAC 802.11 as remote Ethernet for information connect layer qualities, UDP as transport layer attributes and CBR as application layer attributes stay settled

\section{Performance Metrics}

The performance metrics helps to characterize the network that is substantially affected by the routing algorithm to achieve the required Quality of Service (QoS). In this work, the following metrics are considered.

(i). End-to-End Delay(EED): It is the time taken for an entire message to completely arrive at the destination from the source. Evaluation of end-to-end delay mostly depends on the following components

i.e. propagation time (PT), transmission time (TT) queuing time (QT) and processing delay (PD). Therefore, EED is evaluated as:

$$
\mathrm{EED}=\mathrm{PT}+\mathrm{TT}+\mathrm{QT}+\mathrm{PD} .
$$

(ii). Throughput: It is the measure of how fast a node can actually sent the data through a network. So throughput is the average rate of successful message delivery over a communication channel.

(iii). Packet Delivery Ratio (PDR): It is the ratio of the total data bits received to total data bits sent from source to destination.

(iv). Average energy: It is the ratio of total energy consumed by the data packets while transmission to total number of data packets received.

In this work the performance analysis is carried out in an ad-hoc network for four parameters i.e throughput,

End to end delay, average energy and packet delivery ratio(PDR) while keeping other parameters like number of nodes, network area constant. Three protocols i.e. AODV, DSR, and DSDV are considered for the comparison purpose on the above performance.

$\underline{\text { Table 1: simulation parameters for MANETs }}$

\begin{tabular}{|l|l|l|l|}
\hline Simulation parameters & value & Simulation parameters & value \\
\hline Network type & Mobile & Connection type & CBR (constant bit rate) \\
\hline Connection pattern & Random & Simulation area(sq. )m & 1000 \\
\hline Packet size & 1500 bytes & Number of nodes & $10,20,30,40,50$ \\
\hline Duration & $20 \mathrm{~s}$ & Pause time(s) & 0 \\
\hline
\end{tabular}


DOI: https://dx.doi.org/10.26808/rs.ca.i8v2.04

\section{PARAMETERS ANALYSIS}

In this analysis the number of nodes varied from 10 to 50 with an increment of 10 nodes whereas the pause time, network size and simulation duration are fixed at 0s, 1000X1000sqm, and 20s respectively. Other parameters of the network are same as described in the previous section. In simulation, 10 simulation runs for each sample point of a particular protocol generate 10 random scenarios and the average value is used to plot the performance of a network by varying the number of nodes. The performance plots i.e

Delay vs number of nodes (CBR), Throughput vs number of nodes (CBR), Average energy vs number of nodes (CBR), and Packet delivery ratio (PDR) vs number of nodes (CBR) is shown in Fig 1, 2, 3 and 4 respectively

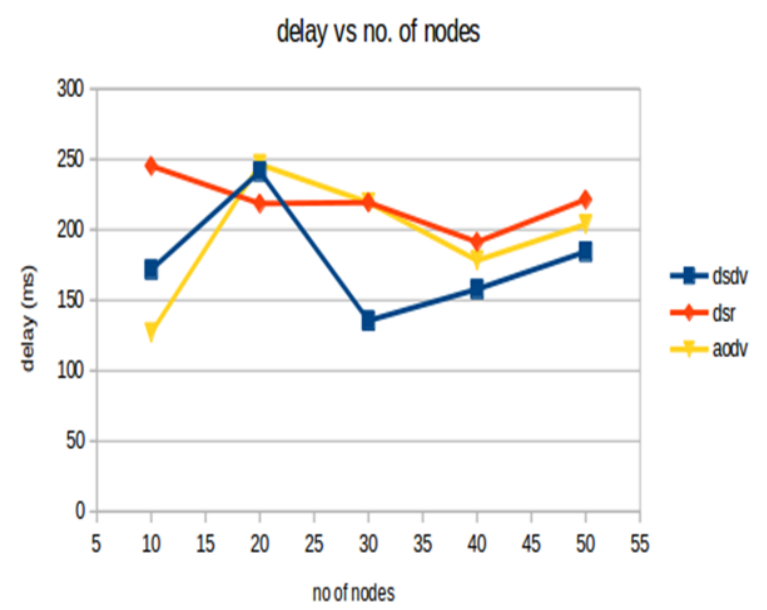

Figure 1: Delay vs number of nodes (CBR).

From figure 1 we observed that delay at lower number of nodes i.e 10 DSR has highest delay followed by DSDV and AODV, at 20 nodes both AODV and DSDV has same delay but this time it is greater than DSR, at 30 nodes DSDV managed to have less delay compared to DSR and a AODV where as these two protocols have same delay, at 40 nodes DSR has greater delay followed by AODV and DSDV and the same scenario followed for higher number of nodes. From this we can conclude that at lower number of nodes AODV has less delay and at higher nodes DSDV has less delay.

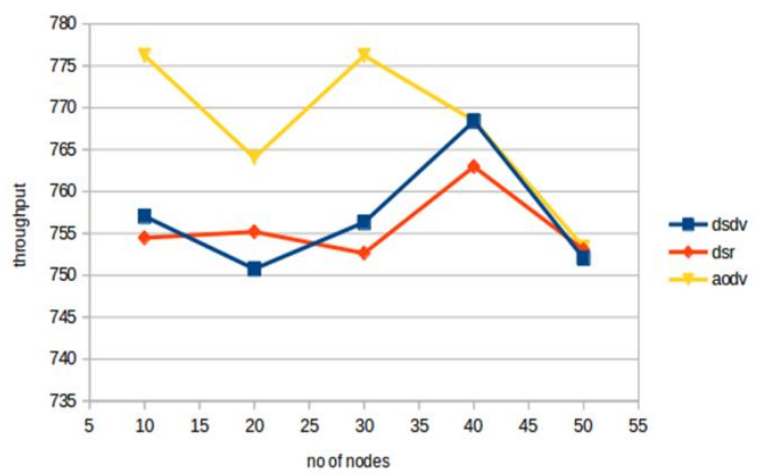

Figure 2: Throughput vs number of nodes 
DOI: https://dx.doi.org/10.26808/rs.ca.i8v2.04 International Journal of Computer Application (2250-1797)

Issue 8 Volume 2, March-April 2018

From figure 2 we observed that the throughput at lower number of nodes i.e 10 nodes AODV has high throughput followed by DSDV and DSR and it is very high compared to DSR and DSDV. For 20 nodes the throughput is decreased compared to throughput for 10 nodes and this time also AODV maintained its position that it has high throughput but DSDV and DSR exchanged their positions. For 30 nodes AODV again has high throughput followed by DSDV and DSR, this time the throughput values are nearly equal to the values at 10 nodes. For 40 nodes AODV and DSDV has equal throughput and DSR has less throughput compared to other two protocols. At 50 nodes all the 3 protocols have same throughput. So overall, at lower nodes i.e below 50 AODV has higher throughput compared to other protocols. At 50 nodes, all the three protocols have same throughput.

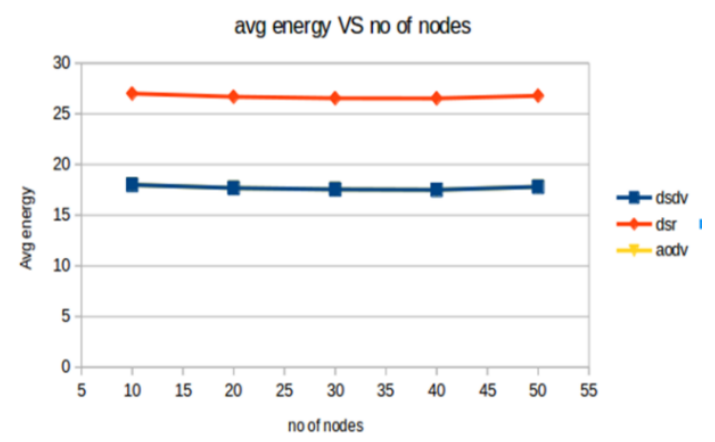

Figure 3: Average energy vs number of nodes

From figure 3 we observed that in terms of average energy consumed AODV and DSR always has same energy for any number of nodes and it is higher than DSDV. Energy consumption of packets delivered through DSDV protocol is always less this is because it uses distance vector technique and frequent updation of routing table, which it will control or stop the formation of loop whenever there is a breakage of transmission path from sender to receiver (sink).

pdr VS no of nodes

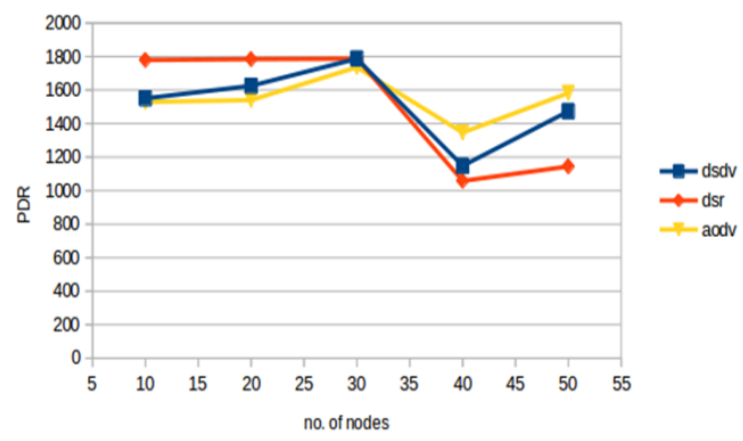

Figure 4: Packet delivery ratio(PDR) vs number of nodes

From figure 4, we observed that PDR for 10 nodes is high for DSR, it is less for AODV and DSDV, both AODV, and DSDV has equal PDR value at 10 nodes. For 20 nodes DSR has same value as for 10 nodes but this time DSDV has over taken the AODV. For 30 nodes all the 3 protocols have same equal PDR. For 40 nodes scenario entirely opposite to that of the scenario at 20 nodes i.e DSR has least PDR, AODV has high and DSDV managed to be in between them. The same scenario is continued further for 50 nodes. So for lower no of nodes DSR has high 
DOI: https://dx.doi.org/10.26808/rs.ca.i8v2.04

PDR and for higher nodes AODV has high PDR. Where DSDV never has high or lower PDR it always managed to be in between the other protocols.

\section{CONCLUSION}

In this paper we assessed the four execution measures i.e. normal vitality, PDR, end-to-end postpone and throughput with various number of hubs by keeping different parameters like interruption time, recreation region, bundle size and length consistent. From comes about announced in segment IV we reasoned that at bring down number of hubs AODV has less deferral and at higher hubs DSDV has less postponement. Additionally for throughput AODV is the best convention among those three for less number of hubs, normal vitality devoured by the DSDV is constantly less for any number of hubs. So DSDV is best convention in thought to normal vitality utilization and for PDR for less number of hubs DSR is the best convention and for higher hubs AODV is the best convention. In future, using these exhibitions we can plan such a convention, to the point that can be reasonably give information uprightness and in addition information conveyance in very arbitrary versatility organize. Our concentration is to break down the vitality measurements as the cost work for directing in these conventions for better QoS applications.

\section{REFERENCES}

[1] Sarkar S, Basavaraju T.G. and Puttamadappa C., “ Ad Hoc Mobile Wireless Networks: Principles, protocols and applications", Auerbach Publications, 2008.

[2] Tonguz $\mathrm{O}$ and Ferrari "Adhoc Wireless Networks-A Communication -Theoritic Perspective, Wiley and Sons”, 2009.

[3] Royer E.M.and Toh C., "A review of current routing protocols for adhoc mobile wireless networks", IEEE personal communications,1999, pp. 46-56.

[4] Tuteja A, Gujral A, Thalia A, "Comparative Performance Analysis of DSDV, AODV and DSR Routing Protocols in MANET using NS2”, IEEE Comp. Society, 2010, pp. 330-333.

[5] Broach J, .Maltz D.A, Johnson D.B, Hu Y and Jetcheva J, "A Performance comparision of Multihop Wireless Ad-Hoc Network Routing Protocols”, ACM MOBICOM, 1998.

[6] Boukhalkhal A, Yagoubi M.B., Djoudi D, Ouinten Y and Benmohammed M, "Simulation of Mobile Adhoc Routing Strategies", IEEE, 2008, pp.128-132.

[7] Perkins C.E. and Royer E.M, Ad-hoc, "On-demand Distance Vector Routing", draft-ietfmanet-aodv-02.txt, 1998.

[8] Perkins C.E and Bhagwat P, "Highly dynamic Destination-Sequenced Distance-Vector Routing (DSDV)for Mobile Computers”, SIGCOMM ACM, 1994, pp. 234-245.

[9] Clausen T, Jacquet P, RFC 3626-“Optimized Link State Routing Protocol (OLSR)”, Oct 2003.

Perkins C.E., Adhoc Networking, Chapter-5, Pearson, US 2000.

[10] Kelvin Fall, "The ns manual (formerly ns Notes \& Documentation)", US Berkerley LBL USC/ISI and Xerox PARC, 2010

[11] Robins A.D.., "GAWK:an effective AWK programming”, 3rd ed, April 2010. 